\title{
Adjustment disorder: current perspectives
}

This article was published in the following Dove Press journal:

Neuropsychiatric Disease and Treatment

\author{
Paulina Zelviene \\ Evaldas Kazlauskas \\ Department of Clinical and \\ Organizational Psychology, Vilnius \\ University, Vilnius, Lithuania
}

\begin{abstract}
Adjustment disorder (AjD) is among the most often diagnosed mental disorders in clinical practice. This paper reviews current status of AjD research and discusses scientific and clinical issues associated with AjD. AjD has been included in diagnostic classifications for over 50 years. Still, the diagnostic criteria for AjD remain vague and cause difficulties to mental health professionals. Controversies in definition resulted in the lack of reliable and valid measures of AjD. Epidemiological data on prevalence of AjD is scarce and not reliable because prevalence data are biased by the diagnostic algorithm, which is usually developed for each study, as no established diagnostic standards for AjD are available. Considerable changes in the field of AjD could follow after the release of the 11th edition of International Classification of Diseases (ICD-11). A new AjD symptom profile was introduced in ICD-11 with 2 main symptoms as follows: 1) preoccupation and 2) failure to adapt. However, differences between the Diagnostic and Statistical Manual of Mental Disorders, Fifth Edition and ICD-11 AjD diagnostic criteria could result in diverse research findings in the future. The best treatment approach for AjD remains unclear, and further treatment studies are needed to provide AjD treatment guidelines to clinicians.
\end{abstract}

Keywords: adjustment disorder, review, diagnosis, prevalence, treatment, DSM, ICD

\section{Introduction}

Adjustment disorder (AjD) is recognized as a stress-response syndrome, which is defined as a maladaptive reaction to an identifiable stressor. ${ }^{1,2}$ The Diagnostic and Statistical Manual of Mental Disorders, Fifth Edition (DSM-5) and the 11th edition of the International Classification of Diseases ${ }^{3}$ (ICD-11) classify AjD along with other stress-related disorders.

There has been a growing interest in AjD over the last decade. However, AjD receives little attention in research. We searched PubMed database for publications on depression and AjD in October 2017. PubMed search in titles and abstracts with the keyword "depression" identified 139,979 research items over the last 10 years. In comparison, search in PubMed with keyword "adjustment disorder" generated only 401 items over the last 10 years. Depression is studied more often in contrast to AjD, based on this preliminary database screening. These findings are surprising because $\mathrm{AjD}$ is diagnosed frequently as major depression (MD), as evidenced from recent health care utilization study of mental disorders in primary health care. ${ }^{4}$ The primary health care register in Sweden revealed 12.4\% prevalence of $\mathrm{MD}$ and $9.2 \%$ prevalence of AjD. ${ }^{4}$ Furthermore, AjD is among the most often diagnosed mental disorders according to the worldwide survey of 4,887 psychiatrists. ${ }^{5}$ More than $50 \%$ of psychiatrists in this study indicated that they use AjD diagnosis at least once per week, along with other mental disorders, such as depressive episode, schizophrenia, bipolar affective disorder, mixed anxiety and depression, or generalized anxiety disorder. ${ }^{5}$ We cannot
Correspondence: Evaldas Kazlauskas Department of Clinical and Organizational Psychology, Vilnius University, Universiteto str 9-202, Vilnius, LT-01513, Lithuania

Email evaldas.kazlauskas@fsf.vu.It $\mathrm{BY}$
hereby accept the Terms. Non-commercial uses of the work are permitted without any further permission from Dove Medical Press Limited, provided the work is properly attributed. For permission for commercial use of this work, please see paragraphs 4.2 and 5 of our Terms (https://www.dovepress.com/terms.php). 
provide an answer as to why AjD is so understudied, despite its high prevalence and frequency in health care. Still, there is a growing body of knowledge relevant for clinicians and researchers about $\mathrm{AjD}$ diagnosis, assessment, prevalence, risk factors, and treatment.

The aim of this paper is to discuss the development of AjD definition in major diagnostic classifications, provide the evidence from studies about AjD, identify current scientific and clinical issues associated with $\mathrm{AjD}$, and discuss future perspectives for the field of $\mathrm{AjD}$ research and clinical practice.

\section{Definition}

$\mathrm{AjD}$ has been recognized in the diagnostic classification systems under related names for $>50$ years. At the time of writing this review, there are 2 official definitions of AjD diagnosis in 2 major diagnostic classifications used in clinical practice: ICD-10 ${ }^{6}$ and DSM-5. ${ }^{7}$ AjD definition is updated in ICD $-11^{3}$ which is to be approved by the World Health Organization (WHO) in 2018. Definition of disorder is important because diagnostic criteria determine empirical research and clinical practice, including development of diagnostic tools and specialized treatments. Despite the similarities between the AjD definitions in DSM-5, ICD-10, and the upcoming ICD-11, important differences are found between classifications and definitions. We discuss AjD definitions and diagnostic criteria in the following section.

\section{$A j D$ definition in DSM}

AjD has gone through a long path of evolution in DSM classification. AjD was recognized under the definition of "transient situational personality disorder" in DSM-I released in 1952. It later transformed into "transient situational disturbances" in DSM-II. ${ }^{9}$ The term "adjustment disorder" for the first time was used in DSM-III in $1980 .{ }^{10} \mathrm{AjD}$ subtypes were added in the following editions of DSM starting from DSM-III. ${ }^{10} \mathrm{AjD}$ in DSM-IV was defined as emotional or behavioral symptoms occurring within 3 months in relation to an identified stressor, reactions of marked distress, and significant impairment in daily functioning. ${ }^{11}$ According to DSM-IV, AjD should not be diagnosed if disturbances meet criteria of another mental disorder or is an exacerbation of preexisting mental disorder, and if symptoms reflect bereavement reactions. The symptoms of AjD in DSM-IV were also limited to the duration of 6 months after the termination of stressor. ${ }^{11}$ AjD subtypes in DSM-IV were as follows: with depressed mood, with anxiety, with mixed anxiety and depressed mood, with disturbance of conduct, and with mixed disturbance of emotions and conduct. ${ }^{11}$

No significant changes in the definition of $\mathrm{AjD}$ diagnostic criteria have been introduced in DSM-5. ${ }^{7}$ The major change was placement of AjD diagnosis in the section of "Trauma- and Stressor-Related Disorders" along with other stress-related disorders, including the reactive attachment disorder, disinhibited social engagement disorder, posttraumatic stress disorder (PTSD), and acute stress disorder. Traumatic or stressful event is an essential diagnostic criterion for each of the disorders recognized in this section. ${ }^{7}$ Also, AjD specifier of acute/chronic defined among the main diagnostic criteria in DSM-IV was not included in DSM-5 $\mathrm{AjD}$ main criteria, but was moved to the description of the diagnosis in DSM-5.

Five basic diagnostic criteria of $\mathrm{AjD}$ are presented in DSM-5. The first criterion indicates that AjD might only be diagnosed if symptoms occurred within 3 months in the context of identifiable stressor(s). The second criterion specifies clinical significance of AjD symptoms meaning that stress reactions should be out of proportion to the normal reactions of the identified stressor according to the social or cultural context, and there should be significant disturbances in important areas of life. The last 3 criteria point out that 3 ) the disturbance should not meet criteria or represent a worsening condition of another mental disorder; 4) AjD should not be considered in cases of normal bereavement reactions; and 5) AjD has a tendency to dissipate during 6 months after the stressor has ended. ${ }^{7}$ The specification of subtypes is included in DSM-5 AjD diagnosis: with depressed mood, with anxiety, with mixed anxiety and depressed mood, with disturbance of conduct, and with mixed disturbance of emotions and conduct. ${ }^{7}$ High risk for suicidality was also identified as an important diagnostic feature for AjD in DSM-5. Issues concerning comorbidity have been resolved by specifying that AjD can be diagnosed when other mental disorder does not explain occurrence of the symptoms related to the stressor. $\mathrm{AjD}$ can also be diagnosed in addition to the most mental disorders. ${ }^{7}$ It is important to note that DSM-5 AjD diagnostic criteria and the subtypes in their essence were not different from DSM-IV definition. These decisions were taken because $\mathrm{AjD}$ was insufficiently researched and no sufficient empirical proof was found for changes to be included in DSM-5.,12 Moreover, several authors also have argued that $\mathrm{AjD}$ was deliberately created to be nonspecific so that it could allow identification of early stages of major psychiatric disorders. ${ }^{2,8} \mathrm{AjD}$ utility was also recognized in 
cases marking serious distress or suicidality, ${ }^{2}$ because being suicidal seems to be "hidden" in AjD category and suicides are much missed in the DSM system.

\section{AjD definition in ICD}

For the first time, AjD was included in the 8th edition of ICD in 1965 under the name of "transient situational disturbances". It was updated to an "adjustment reaction" in ICD-9 in 1975. The ICD-10 AjD definition under the name of "adjustment disorders" is presented in the "Reaction to severe stress, and adjustment disorders" section along with acute stress reaction, PTSD, and other reactions to severe stress and reaction to severe stress, unspecified. ${ }^{6}$ This section is different from others in the ICD-10, because it includes disorders characterized not only by the symptoms but also by exceptionally stressful life event or a significant life change, which causes maladaptation. AjD in ICD-10 is defined as a maladaptive reaction to identified stressor, including significant life change or stressful event. Manifestations of $\mathrm{AjD}$ are characterized by subjective distress and emotional disturbance, and may also include depression, anxiety, and inability to cope symptoms. ${ }^{6}$

A new $\mathrm{AjD}$ symptom structure was introduced for ICD-11 based on the previous empirical findings and available psychological theories of stress-response syndromes ${ }^{13}$ in 2013. The essence of the proposal was identification of the 2 AjD symptoms as follows: 1) preoccupation with stressor and 2) failure to adapt, which interferes with everyday functioning. ${ }^{1}$ The key points of ICD-11 AjD definition are as follows: identifiable stressor(s), maladaptive reactions that occur within 1 month after exposure to stressor and tend to resolve within 6 months if the stressor has ended, symptoms of preoccupation and failure to adapt related with the identified stressor; it was also specified that symptoms do not justify another mental or behavioral disorder. ${ }^{3}$ Major update in the definition of AjD for the ICD-11 was introduction of the new specific symptom structure.

\section{Debates about AjD definition in ICD and DSM}

The ICD-10 and DSM-IV/DSM-5 AjD definitions attracted considerable criticism. Several authors pointed out that the definition itself is rather loose. ${ }^{13}$ This resulted in misuse of the diagnosis in clinical practice. ${ }^{14}$ Mental health professionals meet patients with AjD often, but it is difficult to use this diagnosis in a clinical practice. For example, the analysis of 2,155 psychologists from 23 countries showed that about $40 \%$ of them had a patient with ICD-10 or DSM-IV AjD diagnosis at least once per week, but they also indicated that ICD-10 AjD had low ease of use. ${ }^{15}$

Because of the diagnostic challenges, findings about AjD prevalence are inconsistent across various studies. ${ }^{12} \mathrm{AjD}$ was neglected in major epidemiological studies and AjD research was criticized for poor methodological design. ${ }^{16}$ Furthermore, the existing AjD diagnosis definitions raised constant debates about underdiagnosing AjD and pathologization of normal stress reactions. ${ }^{17}$ It was also proposed that health care professionals tend to use $\mathrm{AjD}$ diagnosis as a residual category, when the symptom profile does not match any other diagnosis. ${ }^{13}$

\section{Assessment}

$\mathrm{AjD}$ is one of the most under-researched psychiatric disorders. ${ }^{2,13}$ The lack of studies is associated with limited resources of valid and reliable measures of AjD. Vague definition of AjD in DSM and ICD hindered the development of AjD diagnostic tools. Currently, no established standards of diagnosing AjD exist based on DSM and ICD diagnostic criteria. Moreover, measures developed for other conditions and disorders are often applied in diagnosing $\mathrm{AjD}$, such as the Clinician Administered PTSD Scale, MINI-International Neuropsychiatric Interview 5.5, WHO quality of life measure Life-BREF for measuring DSM-5 AjD,${ }^{16}$ or the Hamilton Anxiety Rating Scale, Sheehan Disability Scale, and the Montgomery-Asberg Depression Rating Scale for diagnosing DSM-IV AjD. ${ }^{18}$

Differences are found in AjD diagnostic criteria in DSM and ICD. Therefore, clinicians and researchers must be aware of them for taking into account the diagnostic classification to be used for diagnosing AjD. DSM-5 did not present any major changes from DSM-IV AjD diagnostic criteria, except acknowledgement of AjD along with other stress-related disorders. AjD measures developed for DSM-IV diagnostic criteria could be applied in diagnosing AjD using DSM-5. Clinicians or researchers who use ICD must be aware of significant differences between ICD-10 and ICD-11 AjD definitions. AjD definition in ICD-11 has new pattern of symptoms. So, diagnostic measures that were used for ICD-10 AjD diagnosis are not applicable when using ICD-11 for the measurement of $\mathrm{AjD}$ symptoms.

In response to the need for new measures of $\mathrm{AjD}$, the self-report AjD New Module (ADNM) scale for measuring AjD symptoms based on ICD-11 definition was introduced recently. ${ }^{19,20}$ The ADNM-20 consists of 2 parts, which are as follows: 1) the list of the stressors and 2) AjD symptom item list. The second part of the ADNM-20 is composed 
of 20 symptom items identifying reactions to the identified stressors in the first part of the questionnaire, and in addition to the preoccupation and failure to adapt, also measure depressive mood, anxiety, avoidance, and impulsivity. Studies have reported good psychometric properties of the ADNM-20. Internal consistency of the ADNM-20 subscales ranged from Cronbach's $\alpha=0.74$ to $0.90^{19}$ and from Cronbach's $\alpha=0.81$ to 0.85 in other study. ${ }^{21}$ The test-retest reliability of the ADNM-20 subscales was high, ranging from 0.61 to $0.84 .{ }^{19}$ A study that investigated burglary victims reported high internal consistency for the total scale of the ADNM-20 with Cronbach's $\alpha=0.94$, and internal consistency for the subscales of ADNM-20 ranged from Cronbach's $\alpha=0.80$ to $0.89 .^{20}$ The internal consistency of Lithuanian version of the ADNM-20 subscales was good with Cronbach's $\alpha$ ranging from 0.65 to $0.87 .^{22}$

The ADNM is providing a new promising approach for measuring ICD-11 AjD, but this measure is still in development. There are several versions of the ADNM scale available, including a brief 8-item ADNM-8 scale. ${ }^{23,24}$ Diagnostic algorithm for AjD diagnosis was proposed in several studies. ${ }^{20,25}$ However, further studies are needed to provide clinical utility of this self-report measure in clinical practice.

\section{Prevalence}

$\mathrm{AjD}$ is one of the most often used diagnoses in clinical practice. $^{1,2,5,15}$ However, data on prevalence of AjD is very limited due to the lack of studies. Moreover, epidemiological data of AjD are limited because AjD was not included in major national health surveys. ${ }^{16}$

The available studies show $1 \%-2 \%$ prevalence of $\mathrm{AjD}$ in the general population. Based on ICD-10 criteria and using Schedule for Clinical Assessment in Neuropsychiatry for diagnosis of $\mathrm{AjD}$, the prevalence in the general population of $\mathrm{AjD}$ ranged from $0.2 \%$ to $1 \%$ in a multinational study conducted in Finland, Ireland, Norway, and Spain. ${ }^{26}$ The prevalence of $\mathrm{AjD}$ was found to be $0.9 \%-2 \%$ in a representative study from Germany $(\mathrm{N}=2,512)$ based on the ICD-11 criteria using various AjD diagnostic algorithms developed for the ADNM-20. ${ }^{25,27}$ The prevalence of AjD among elderly in Switzerland $(\mathrm{N}=570)$ was found to be $2.3 \%$ using ADNM. ${ }^{14}$

In clinical and high-risk samples, AjD prevalence was found to be higher compared with general population studies. A study of primary health care in Spain $(\mathrm{N}=3,815)$ found $2.9 \%$ prevalence of AjD using Structured Clinical Interview for DSM-IV Axis I Disorders. ${ }^{28}$ Swedish study of Primary Care Registry for exploration of common psychiatric disorders in primary care setting showed that $\mathrm{AjD}$ was one of the most prevalent disorders (9.2\%) along with MD (12.4\%) and anxiety disorders (9.9\%). ${ }^{4}$ A recent longitudinal study of individuals after major injury $(\mathrm{N}=826)$ in Australia found AjD prevalence of $18.9 \%$ at 3-month and $16.3 \%$ at 12 -month follow-up based on DSM-5 criteria for AjD.

The current DSM-5 and ICD-11 AjD definitions are based on exclusion criteria, and if an individual is diagnosed with another mental disorder, AjD diagnosis should be excluded. However, there is a growing interest in exploring association of $\mathrm{AjD}$ with other mental disorders. Individuals diagnosed with $\mathrm{AjD}$ had a $3.7 \%$ probability for the diagnosis of $\mathrm{MD}$, and $3.0 \%$ probability for anxiety disorders in a recent health care utilization study in Sweden. ${ }^{4}$ AjD had high correlations with $\mathrm{MD}(r=0.53)$ and anxiety disorders $(r=0.49) .{ }^{4}$ The other study in Israel explored association between the PTSD and AjD recently. ${ }^{29}$ This study found that previous exposure to stressful events during the past month was not significant predictor for PTSD unlike previous exposure to trauma, but both stressor factors were significant predictors for AjD. Furthermore, physical proximity related to trauma exposure was also a significant factor for PTSD, but not for AjD. ${ }^{29}$

\section{Theoretical conceptualization of $\mathrm{AjD}$}

Theoretical framework of $\mathrm{AjD}$ is another major challenge in this field. AjD is often viewed as a transient condition between the normal and pathological condition. Strain and Diefenbacher ${ }^{8}$ have argued that usefulness of $\mathrm{AjD}$ for the practitioners lies in its position between normality and pathology, as AjD has been acknowledged as subthreshold diagnosis. Furthermore, the current AjD symptom profile in DSM allows recognition of early or temporary mental states that did not reach all diagnostic criteria of other major psychiatric disorder. ${ }^{2,13}$

Despite the ongoing debates about the diagnostic challenges of $\mathrm{AjD}$, it was only very recently that the first theoretical conceptualization of AjD was proposed. ${ }^{13}$ Maercker et $\mathrm{al}^{13}$ conceptualized $\mathrm{AjD}$ as a stress-response syndrome, and proposed the new diagnostic criteria based on the psychological stress-response theories, and tested their proposals empirically in a clinical sample. ${ }^{13}$ Since AjD was proposed to be viewed as a stress-response syndrome explaining human reactions following stressful events, PTSD theoretical background and research data were used in order to make assumptions about psychological mechanisms of AjD.

The main difference between AjD and PTSD is the intensity of a stressful event, which can lead to qualitatively 
different stress responses. Stressors that are linked to AjD usually do not have a sudden and unexpected threat to human life, and are not as intense as traumatic events in the case of PTSD. ${ }^{13,29}$ Examples of life stressors that are associated with AjD could be divorce, illness or disability, financial problems, conflicts with co-workers, relocation, and retirement, among others. ${ }^{13}$ The assumption that $\mathrm{AjD}$ is a non-adaptive response to clearly identifiable stressors has referred to psychological theories that explain posttraumatic stress and provide an initial theoretical justification. ${ }^{13}$

Application of psychological theories of PTSD is limited for conceptualization of AjD symptoms. PTSD and AjD differ in the nature of stressor and symptoms. However, use of the theories of PTSD is justified by the fact that AjD is placed under stress-related chapters in DSM-5 and ICD-11. Furthermore, we know that the role of psychological theories in the field of psychotraumatology is significant. These psychological theories facilitated the development of effective evidence-based treatment for PTSD, such as trauma-focused cognitive behavioral therapy. Theories of AjD could guide research and contribute to the development of new psychosocial treatments for AjD. However, more research would be needed not only from psychosocial studies but also from neuroscience and genetic or epigenetic studies to provide data for a better understanding of AjD.

\section{Perspectives}

Definitions of AjD in DSM-IV/DSM-5 and ICD-10 have received considerable criticism. ${ }^{8,12,13,30} \mathrm{AjD}$ definitions are inaccurate, raising doubts about their validity. ${ }^{12,17}$ The disorder still maintains a subthreshold status, which means that $\mathrm{AjD}$ cannot be diagnosed if diagnostic threshold of other disorder is reached. No clear diagnostic AjD criteria exist, stressors are not clearly defined, and subtypes of AjD in DSM are questionable. ${ }^{30}$ Furthermore, there is not enough research to provide evidence about distinction of $\mathrm{AjD}$ from depressive or other mental disorders. Diagnosis of AjD in clinical practice and research is complicated due to the lack of measures and needs to be addressed in future research.

The current state of AjD diagnostic criteria in DSM-5 and updates presented in ICD-11 might be a significant incentive for future research in the field. Debates are still ongoing about the validity of $\mathrm{AjD}$ symptom profile, and there is a tremendous need for research that would help to clarify AjD diagnostic criteria. ${ }^{16}$ Recognizing AjD along with other disorders that are associated with stress and making stressor(s) criteria as a mandatory condition for diagnosing $\mathrm{AjD}$ gave new perspectives for its theoretical assumptions and future research. Another significant development was the specification of AjD symptoms in ICD-11, although this path has to be more evidence-based in the future research. Since ICD-11 identifies 2 symptoms of preoccupation and failure to adapt, and DSM-5 AjD is defined by a broader definition of emotional and behavioral symptoms, considerable differences could rise in DSM vs ICD diagnosis, measures, and research in the future. This could be a barrier for further advancement of the AjD field.

Probably, the most common obstacle for the development of $\mathrm{AjD}$ field was criticism for pathologizing normal reactions to stress. All humans are exposed to life stressors at one time or another in their lives, and react to stressors. AjD could be understood by using an analogy of "flu", which is characterized as an infectious respiratory system disease caused by influenza virus. "Flu" is diagnosed through symptoms, such as fever, cough, and headache among others. Majority of patients recover; however, some individuals may develop serious complications because of flu. AjD, in this analogy, could be assumed as sort of "mental flu". Research indicates that majority of the population are exposed to life stressors regularly. Humans react to stressors and the majority are capable of coping with these stressors. However, some individuals might have various mental disorders associated with stress. AjD might represent a stress-response reaction and condition, which needs to be acknowledged following an identifiable stressor as maladaptive reaction. Health care services for individuals diagnosed with AjD should be provided in order to avoid serious complications and other mental disorders in the future. However, we could also expect many individuals with $\mathrm{AjD}$ to recover without treatment, as people have inner resources and social support that could significantly contribute to coping with life stressors and AjD symptoms.

The analysis of risk factors in research of AjD might provide better understanding of $\mathrm{AjD}$. There is some evidence that female gender might be one of the risk factors for AjD. ${ }^{4}$ The same study also found that AjD was associated with increased probability for divorce. ${ }^{4}$ There is growing evidence that AjD could be associated with stressful life events, and might occur following exposure to traumatic events, such as terror attack ${ }^{29}$ or serious injury. ${ }^{16}$ Previous traumatic events could be a risk factor for AjD. One of the first studies based on ICD-11 criteria for $\mathrm{AjD}$ found that previous exposure to stressful or traumatic events during the last month was significantly associated with current symptoms of ICD-11 AjD. ${ }^{29}$

Little is known about the course of AjD over time. One of the first longitudinal studies in adult sample have identified that, contrary to the definition, AjD can be persistent 
over time. AjD was highly prevalent among survivors of major injury even 12 months after traumatic event. ${ }^{16}$ However, this study did not include patients with the suicide risk, which could eliminate a significant proportion of $\mathrm{AjD}$ cases from the study, ${ }^{16}$ as previous studies indicated links between AjD and suicidality. ${ }^{31}$ Still, suicidality is not included in a majority of AjD studies, and even the newly proposed ADNM measure does not have suicide risk screening items. Longitudinal studies are needed to identify the trajectories of AjD symptoms, risk, and protective factors in the future, especially among children and adolescents.

There is little evidence about the effectiveness of psychopharmacological or psychosocial treatments for AjD. Psychological treatments for PTSD are the most effective, ${ }^{32,33}$ and generally regarded as the first choice of treatment for PTSD. We could predict that AjD, as a stress-response reaction, could be successfully treated with psychosocial interventions as well. Development and validation of evidence-based AjD psychosocial treatments is very important for clinicians. However, due to diversity of stressful experiences, it might be difficult to develop and test universal interventions that would fit in each particular stressor case. Still, there are the first attempts to develop low-intensity cost-effective self-help interventions for AjD. ${ }^{34,35}$ Internetbased interventions could be effective for the treatment of AjD. ${ }^{23}$ More research is needed to implement evidence-based effective AjD treatments in clinical practice.

\section{Acknowledgment}

This research was funded by the European Social Fund under the number 09.3.3-LMT-K-712-02-0096 “Development of Competences of Scientists, other Researchers and Students through Practical Research Activities" measure.

\section{Disclosure}

The authors report no conflicts of interest in this work.

\section{References}

1. Maercker A, Brewin CR, Bryant RA, et al. Diagnosis and classification of disorders specifically associated with stress: Proposals for ICD-11. World Psychiatry. 2013;12(3):198-206.

2. Strain JJ, Friedman MJ. Considering adjustment disorders as stress response syndromes for DSM-5. Depress Anxiety. 2011;28(9):818-823.

3. World Health Organization. ICD-11 Beta Draft. Available from: https:// icd.who.int/dev11/1-m/en. 2017. Accessed October 1, 2017.

4. Sundquist J, Ohlsson H, Sundquist K, Kendler KS. Common adult psychiatric disorders in Swedish primary care where most mental health patients are treated. BMC Psychiatry. 2017;17(1):235.

5. Reed GM, Mendonça Correia J, Esparza P, Saxena S, Maj M. The WPAWHO global survey of psychiatrists' attitudes towards mental disorders classification. World Psychiatry. 2011;10(2):118-131.

6. World Health Organization. The ICD-10 Classification of Mental and Behavioural Disorders. Geneva: World Health Organization; 1992.
7. American Psychiatric Association. Diagnostic and Statistical Manual of Mental Disorders, 5th Edition (DSM-5). Washington, DC: American Psychiatric Association; 2013.

8. Strain JJ, Diefenbacher A. The adjustment disorders: the conundrums of the diagnoses. Compr Psychiatry. 2008;49(2):121-130.

9. American Psychiatric Association. Diagnostic and Statistical Manual of Mental Disorders: DSM-II. Washington, DC: American Psychiatric Publications; 1968.

10. American Psychiatric Association. Diagnostic and Statistical Manual of Mental Disorders (3rd ed), (DSM-III). Washington, DC: American Psychiatric Association; 1980.

11. American Psychiatric Association. Diagnostic and Statistical Manual of Mental Disorders (4th ed) (DSM-IV). Washington, DC: American Psychiatric Association; 1994.

12. Semprini F, Fava GA, Sonino N. The spectrum of adjustment disorders: too broad to be clinically helpful. CNS Spectr. 2010;15(6):382-388.

13. Maercker A, Einsle F, Köllner V. Adjustment disorders as stress response syndromes: a new diagnostic concept and its exploration in a medical sample. Psychopathology. 2007;40(3):135-146.

14. Maercker A, Forstmeier S, Enzler A, et al. Adjustment disorders, posttraumatic stress disorder, and depressive disorders in old age: findings from a community survey. Compr Psychiatry. 2008;49(2):113-120.

15. Evans SC, Reed GM, Roberts MC, et al. Psychologists' perspectives on the diagnostic classification of mental disorders: results from the WHO-IUPsyS Global Survey. Int J Psychol. 2013;48(3):177-193.

16. O'Donnell ML, Alkemade N, Creamer M, et al. A longitudinal study of adjustment disorder after trauma exposure. Am J Psychiatry. 2016; 173(12):1231-1238.

17. Casey P, Dowrick C, Wilkinson G. Adjustment disorders: fault line in the psychiatric glossary. Br J Psychiatry. 2001;179:479-481.

18. Bachem R, Perkonigg A, Stein DJ, Maercker A. Measuring the ICD-11 adjustment disorder concept: validity and sensitivity to change of the adjustment disorder - new module questionnaire in a clinical intervention study. Int J Methods Psychiatr Res. 2017;26(4):1-9.

19. Einsle F, Köllner V, Dannemann S, Maercker A. Development and validation of a self-report for the assessment of adjustment disorders. Psychol Health Med. 2010;15(5):584-595.

20. Lorenz L, Bachem RC, Maercker A. The adjustment disorder-new module 20 as a screening instrument: cluster analysis and cut-off values. Int J Occup Environ Med. 2016;7(4):215-220.

21. Bley S, Einsle F, Maercker A, Weidner K, Joraschky P. Evaluation of a new concept for diagnosing adjustment disorders in a psychosomatic setting. Psychother Psychosom Medizinische Psychol. 2008; 58(12):446-453.

22. Zelviene P, Kazlauskas E, Eimontas J, Maercker A. Adjustment disorder: empirical study of a new diagnostic concept for ICD-11 in the general population in Lithuania. Eur Psychiatry. 2017;40:20-25.

23. Eimontas J, Gegieckaite G, Dovydaitiene M, et al. The role of therapist support on effectiveness of an internet-based modular self-help intervention for adjustment disorder: a randomized controlled trial. Anxiety Stress Coping. Epub 2017 Oct 6.

24. Horn $\mathrm{AB}$, Maercker A. Intra- and interpersonal emotion regulation and adjustment symptoms in couples: the role of co-brooding and co-reappraisal. BMC Psychol. 2016;4(1):51.

25. Glaesmer H, Romppel M, Brähler E, Hinz A, Maercker A. Adjustment disorder as proposed for ICD-11: dimensionality and symptom differentiation. Psychiatry Res. 2015;229(3):940-948.

26. Casey P, Maracy M, Kelly BD, et al. Can adjustment disorder and depressive episode be distinguished? Results from ODIN. J Affect Disord. 2006;92(2-3):291-297.

27. Maercker A, Forstmeier S, Pielmaier L, Spangenberg L, Brahler E, Glaesmer H. Adjustment disorders: prevalence in a representative nationwide survey in Germany. Soc Psychiatry Psychiatr Epidemiol. 2012;47(11):1745-1752.

28. Ventevogel P, Pérez-Sales P, Férnandez-Liria A, Baingana F. Integrating mental health care into existing systems of health care: during and after complex humanitarian emergencies. Interv Int J Ment Heal Psychosoc Work Couns Areas Armed Confl. 2011;9(3):195-210. 
29. Mahat-Shamir M, Ring L, Hamama-Raz Y, et al. Do previous experience and geographic proximity matter? Possible predictors for diagnosing adjustment disorder vs. PTSD. Psychiatry Res. 2017;258:438-443.

30. Casey P. Adjustment disorder: new developments. Curr Psychiatry Rep. 2014;16(6):451

31. Casey P, Jabbar F, O'Leary E, Doherty AM. Suicidal behaviours in adjustment disorder and depressive episode. J Affect Disord. 2015;174: 441-446.

32. Bisson JI, Roberts NP, Andrew M, Cooper R, Lewis C. Psychological therapies for chronic post-traumatic stress disorder (PTSD) in adults. Cochrane Database Syst Rev. 2013;12:CD003388.
33. Courtois CA. Complex trauma, complex reactions: assessment and treatment. Psychol Trauma Theory Res Pract Policy. 2008;S(1):86-100.

34. Skruibis P, Eimontas J, Dovydaitiene M, Mazulyte E, Zelviene P, Kazlauskas E. Internet-based modular program BADI for adjustment disorder: protocol of a randomized controlled trial. BMC Psychiatry. 2016;16(1):264.

35. Bachem R, Maercker A. Self-help interventions for adjustment disorder problems: a randomized waiting-list controlled study in a sample of burglary victims. Cogn Behav Ther. 2016;45(5):397-413.

\section{Publish your work in this journal}

Neuropsychiatric Disease and Treatment is an international, peerreviewed journal of clinical therapeutics and pharmacology focusing on concise rapid reporting of clinical or pre-clinical studies on a range of neuropsychiatric and neurological disorders. This journal is indexed on PubMed Central, the 'PsycINFO' database and CAS, and is the official journal of The International Neuropsychiatric Association (INA). The manuscript management system is completely online and includes a very quick and fair peer-review system, which is all easy to use. Visit http://www.dovepress.com/testimonials.php to read real quotes from published authors.

Submit your manuscript here: http://www.dovepress.com/neuropsychiatric-disease-and-treatment-journal 